\title{
Investigation on the Effects of Intake Grill in the Performance of Cooling Module Under Vehicle Working Conditions
}

\author{
He Chang ${ }^{*}, 1,2$, Xiumin YU² and Jing Hua Lv ${ }^{1}$ \\ ${ }^{I}$ FAW CAR Co. Ltd. Chang Chun, China \\ ${ }^{2}$ State Key Laboratory of Automotive Simulation and Control, Jilin University, Changchun, China
}

\begin{abstract}
Air path model of cabin for cooling module simulation for vehicle has been constructed according to the test, and the simulation results were compared with the test data to verify the authenticity of the module. This simulation model provides an effective platform for vehicle thermal management analysis. The module is used to simulate the different intake grills and to find how different grills affect the performances of the cooling module. The intake grill effect significantly influences the performance of airflow into the cabin.
\end{abstract}

Keywords: Cooling module, Intake grill, Simulation, Vehicle.

\section{INTRODUCTION}

The shape of the front car on one hand, determines the airflow of cabin and pressure effective for the frontal area, and on the other hand, it is the starting point for interior flow field of the cabin. Therefore, it affects the aero-dynamics of the cabin. As it is known, the internal flow situation of the cabin is extremely complex, and subtle changes in structural characteristics of the entire flow field have a great impact on the cooling module. However, the cooling module of the cabin makes the work environment complex [1-3].

\section{SIMULATION}

\subsection{Engine Input Dates}

The parameter of engine is used as shown in Table 1. It is determined by the heat balance test and the drag test in vehicle working for the energy distribution of combustion to measure the temperature of coolant as shown in Fig. (1).

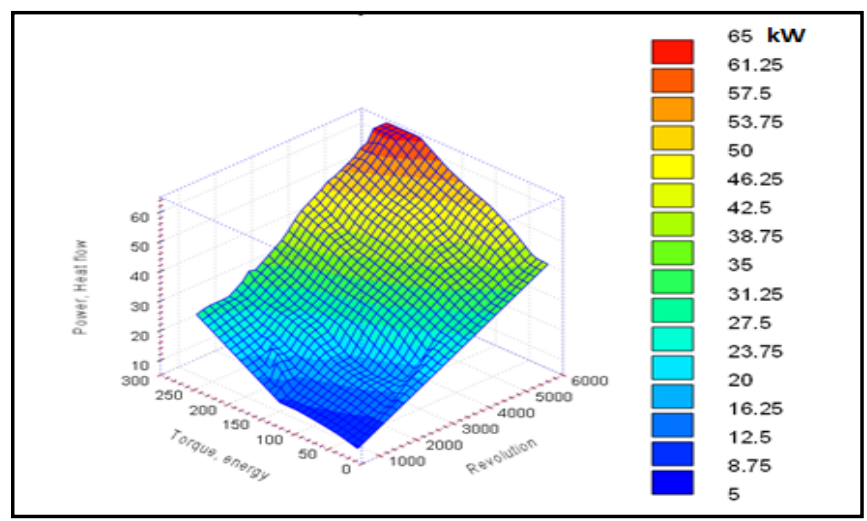

Fig. (1). The engine heat map for cooling system.

\footnotetext{
*Address correspondence to this author at the FAW CAR Co. Ltd. Chang Chun, China; Tel: 0431-13944836786; Fax: 0431-81504449; E-mail:n4n4china@126.com
}

Table 1. The engine main date.

\begin{tabular}{|c|c|}
\hline \multicolumn{2}{|c|}{ Engine Main Date } \\
\hline \hline Displacement: 2.0T & Power: $120 \mathrm{~kW}$ \\
\hline Cylinder: 4 & Rated: $6000 \mathrm{r} / \mathrm{min}$ \\
\hline
\end{tabular}

Cooling module input dates are shown in Table 2.

Table 2. Cooling module input date.

\begin{tabular}{|c|c|}
\hline \multicolumn{2}{|c|}{ Cooling Module Input Date } \\
\hline \hline 1 & Coolant: Water / glycol \\
\hline 2 & The pump curve \\
\hline 3 & Fan characteristic curve \\
\hline 4 & Condenser heat transfer curve \\
\hline 5 & Cooling system pressure \\
\hline 6 & Radiator heat transfer curve \\
\hline 7 & Radiator air resistance curve \\
\hline 8 & Condenser resistance curve \\
\hline 9 & CAC resistance curve \\
\hline 10 & CAC heat transfer curve \\
\hline
\end{tabular}

\subsection{Cabin Air Path Model Input Dates}

By the CFD instead of the wind tunnel, air characteristic parameters are obtained, which help in building the KULI as shown in Figs. $(\mathbf{2}, \mathbf{3})[4,5]$. 


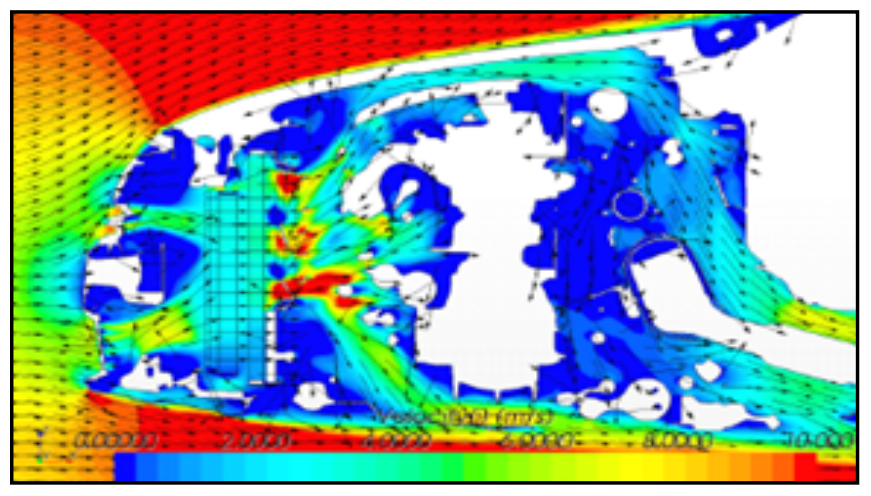

Fig. (2). The cabin CFD result.

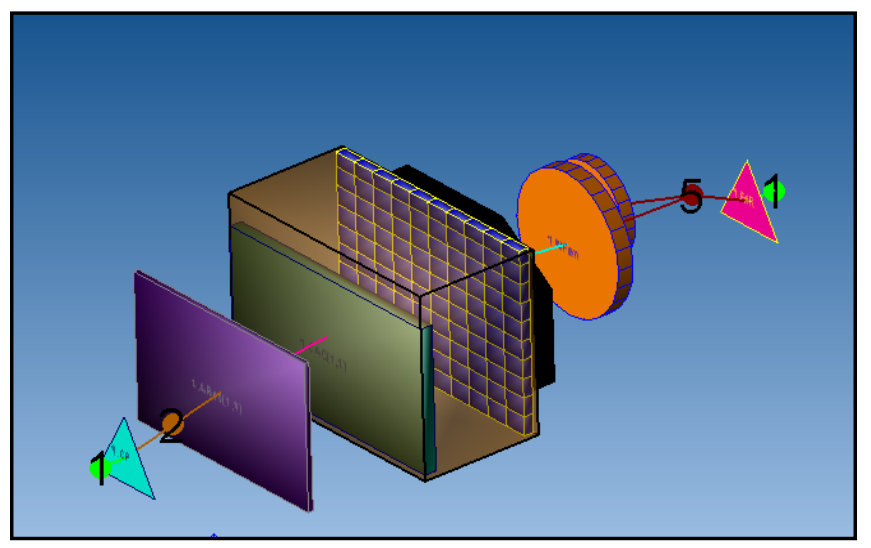

Fig. (3). Air path model of cabin for cooling module.
The model of cooling module for heat balance test is shown in Fig. (4). Steady simulation is maintained at a temperature of $35^{\circ} \mathrm{C}$, with the cooling fan running at full rotation, and the vehicle being at a speed of $40 \mathrm{~km} / \mathrm{h}$.

\section{VERIFICATION AND ANALYSIS}

The simulation results are compared with the test data, as shown in Figs. $(5,6)$ and the error of $5 \%$ within, is caused by the very complex flow field in the cabin. There is heat reflux in a part of the cooling module making simulation difficult. The model for cooling system can predict and analyze the performance of the cooling system.

\subsection{Analysis Intake Boost Coefficient CP}

According to the Bernoulli's equation of fluid mechanics, when the air flows through the grill, suddenly the crosssection becomes smaller $[6,7]$. There is a high-pressure zone near the cabin outside. So, the outside pressure of cabin is higher than the inside pressure. It is much easier for air to flow smoothly into the cabin. This phenomenon is defined as the intake inertia, which expresses an intake boost coefficient $\mathrm{CP}$. The grill surface of measured pressure value is converted to the form of the pressure coefficient $\mathrm{Cp}$.

$\mathrm{CP}=\mathrm{P} /\left(0.5 * \rho * \mathrm{~V}^{2}\right)$

where $P$ is the pressure near the intake grill, $\rho$ is the air density and V is the vehicle speed. Fig. (12) shows the fan load of $100 \%$ signifying the relationship between the boost coefficient $\mathrm{CP}$ and vehicle speed.

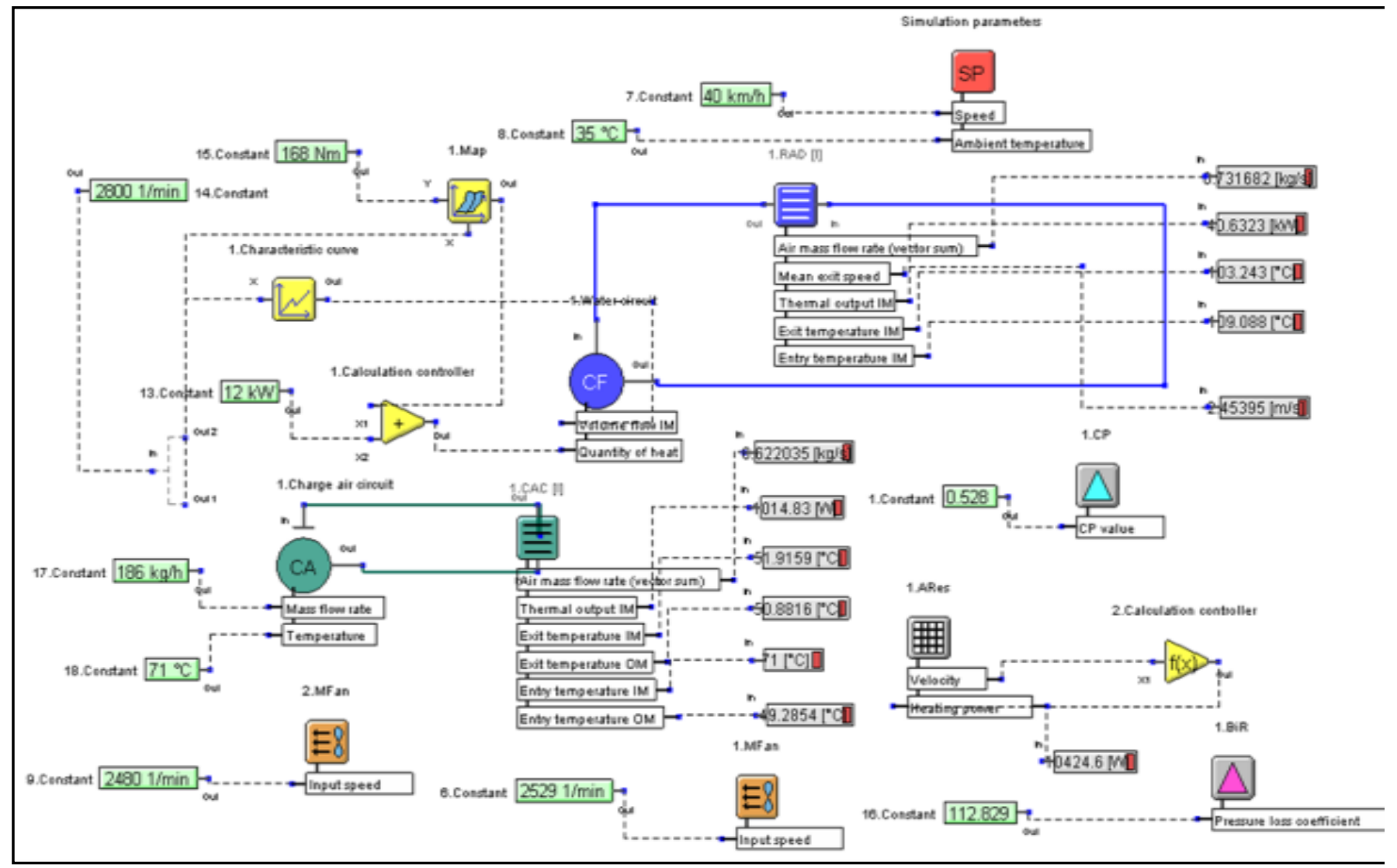

Fig. (4). Cooling module heat transfer model. 


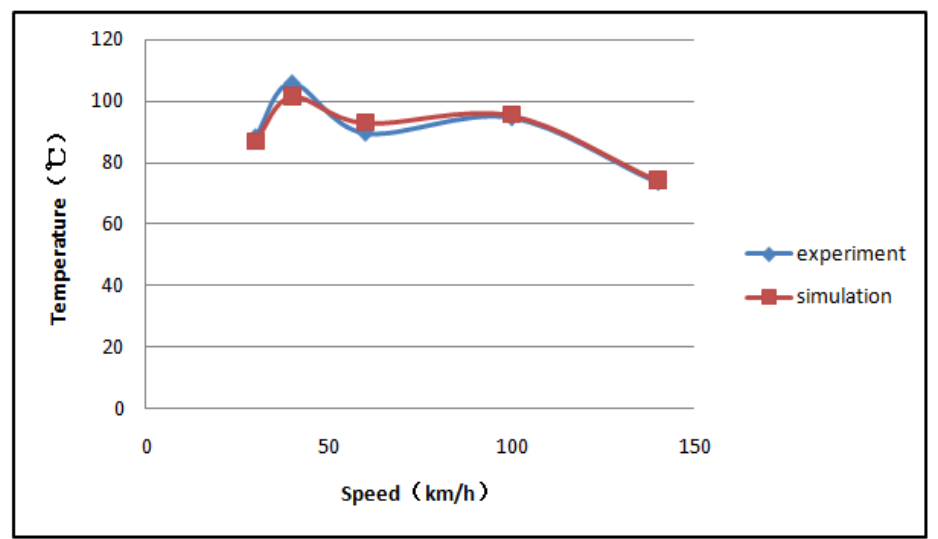

Fig. (5). The coolant of radiator temperature.

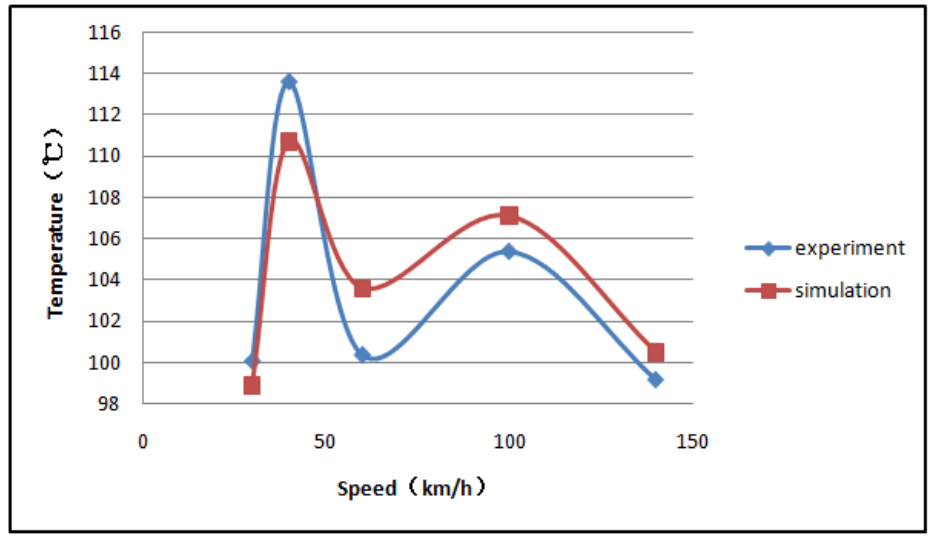

Fig. (6). The coolant of engine temperature.

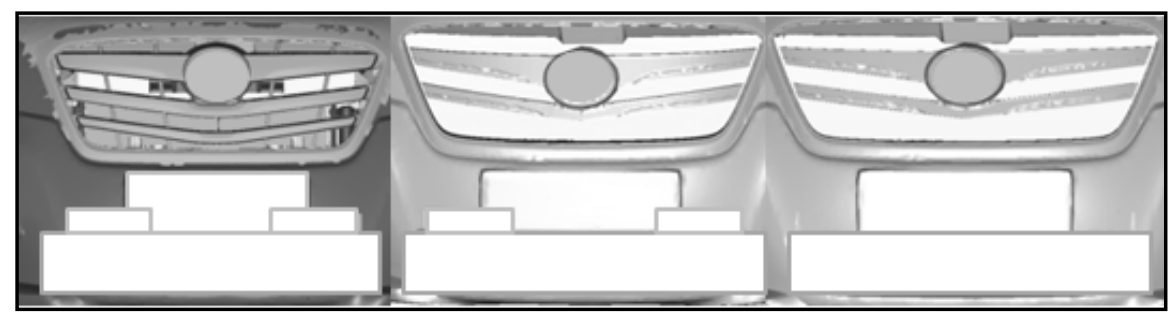

C1:

C2:

C3:

Fig. (7). The different intake grills.

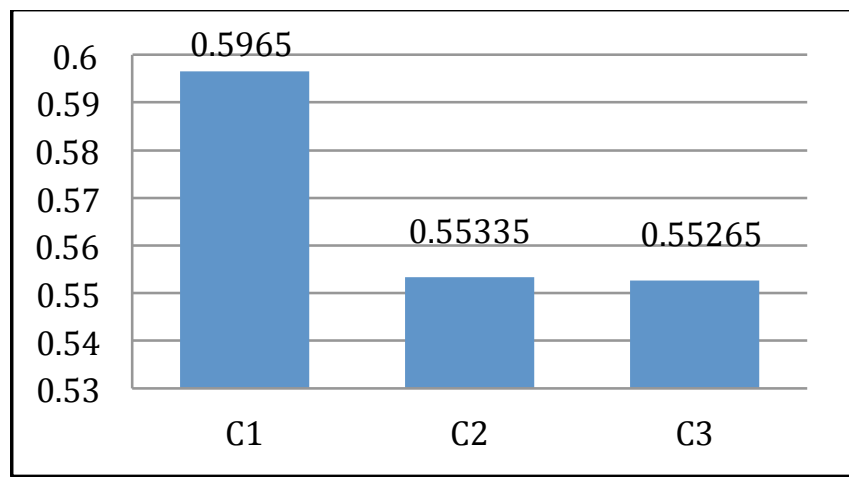

Fig. (8). The intake boost coefficient CP.

This paper focuses on the typical operating conditions of the vehicle at low speed and high torque engine under load on the cooling module performance analysis and calculation of 3 cases of intake grills as shown in Fig. (7).

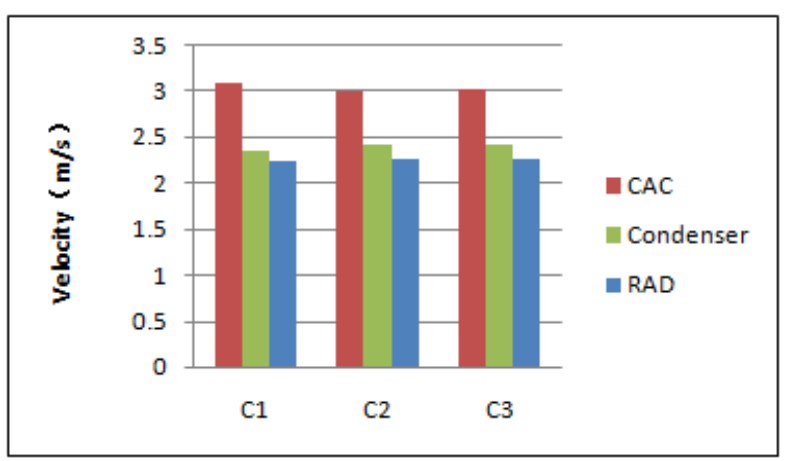

Fig. (9). The intake boost coefficient CP.

By the CFD, the CP is obtained as shown in Fig. (8).

CASE 1: the upper grill to modify the angle of the wind guide.

CASE 2: at lower opening, the upper grill styling changes. 


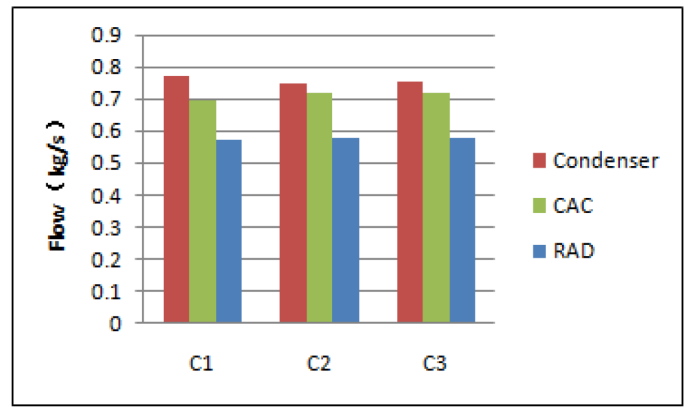

Fig. (10). The intake boost coefficient CP.

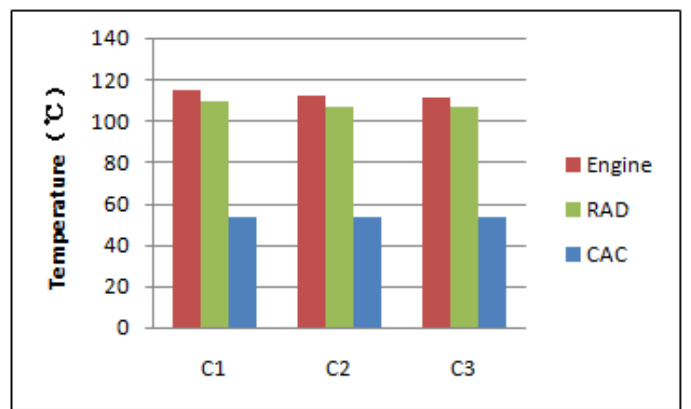

Fig. (11). The intake boost coefficient CP.

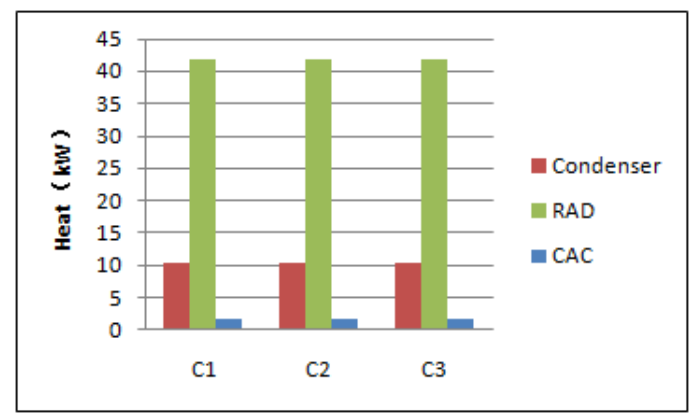

Fig. (12). The intake boost coefficient CP.

CASE 3: being lower without opening, the intake grill and CASE 2 are the same.

\subsection{The Intake Grill effect on the Flow of the Cooling Module}

The analysis of different intake grills for the airflow of cooling module is shown in Figs. $(\mathbf{9}, \mathbf{1 0})$. The simulation data indicates that the different intake grills and the airflow of the cooling system are much sensitive. In case 1, CAC airflow increased by $1.23 \%$ compared with the original value. Radiator airflow improved by $7.35 \%$ of the original value. In case 2 , CAC airflow increased by $1.49 \%$ compared with the original value. Radiator airflow increased by $3.04 \%$ compared with the original value. In case 3 , CAC airflow increased by $1.75 \%$ compared with the original value.
Radiator airflow increased by $3.54 \%$ compared with the original value.

\subsection{The Intake Grill effect on the Heat Transfer of the Cooling Module}

The analysis of different intake grills for the heat transfer of cooling module is shown in Figs. $(\mathbf{1 1}, \mathbf{1 2})$. The data shows that with different shapes of the cooling module front, air intake efficiency has less influence and can affect the heat transfer performance of the cooling module. The $\mathrm{CP}$ of the different grill has a different temperature of CAC, but the heat transferred between RAD and engine is slightly different. Because the grill is the starting point of the flow field inside the cabin, it changes the direction of airflow into the cabin interior. Proper angle of grill and layout can make a reasonable airflow into the cabin, and cooling module needs more airflow to take heat away.

\section{CONCLUSION}

$\mathrm{CP}$ is expressed as the capacity of air into the cabin. The larger the $\mathrm{CP}$, the better is the ability of air to enter the cabin. It is effective to improve the velocity so that the grill can cause greater airflow by changing the angle of the intake grill. The results show that for the car that only changes the shape of the intake grill, the capacity of air into the cabin is different. A different CP leads to different airflows. But the performance of the cooling module has nothing to do with the CP. CP and airflow can be changed by changing the shape of the intake grills and cooling modules to improve the efficiency of heat transfer.

\section{CONFLICT OF INTEREST}

The authors confirm that this article content has no conflict of interest.

\section{ACKNOWLEDGEMENTS}

Declared none.

\section{REFERENCES}

[1] O. Fischer, T. Kuthada, J. Wiedemann, P. Dethioux, R. Mann, and B. Duncan, "CFD validation study for a sedan scale model in an open jet wind tunnel", SAE Paper, vol. 1, pp. 0325, 2008.

[2] S.M. Yang, and T. Wenquan. Heat transfer [M]. Version 4. Higher Education Press: Beijing, 2006, pp. 216-217. (in Chinese).

[3] KULI 8.0 User manual, Magna Styer Engineering.

[4] D. Wei, W. Jack, K. Dinakara, and S. Sandeep. CFD application in automotive front-end design [C], SAE Paper, vol. 1, pp. 0337, 2006.

[5] Wolf-Heinrich Hucho. Aerodynamics of Road Vehicles. SAE book No. R-177, 199.

[6] L. Martinez, L. J. Guerra, G. Ferichola, A. Garcia, J. Yang, and Yao, Stiffness Corridors for the Current European Fleet, Technical Report APSP31-0009R, APROSYS (2006).

[7] J. Larmini, and A. Dicks, "Fuel cell system explainer" [M]. John Wile \& Sons, Inc, England: 2003, pp. 22-24. 